interfere with prescribing alarmed the Australian Medical Association. The association argued that the new proposal did not deal with the government's real problems-the rapid escalation of the cost of free prescriptions for pensioners and the specially subsidised prescriptions for disadvantaged patients. Together these represent almost three quarters of total costs.

As most of these 53 items were not expensive items the move was seen as misguided, and in partial recognition of this the government has already removed 32 items from the new authority list.

The proposals promote unnecessary conflict with doctors. For example, at one point the government proposed that if an authority was shown to be inappropriate it would recoup the costs of the prescription from the doctor. Moreover, the present publicity has probably exaggerated the value of angiotensin converting enzyme inhibitors and may increase their use and costs long term.

Doctors respond well to the authorny system when it is used to avoid adverse drug effects but poorly when it is used to control costs. After a small hiccup they will probably continue their present prescribing practices by increasing the number of authority requests. Moreover, when frustrated by the authority system they will probably turn to the next most expensive alternatives with little eventual savings. If they need to use two of these drugs to achieve the same effect it may even represent an increased cost to the scheme and no benefit to the patient.

The concerns of the pharmaceutical industry about the implementation of generic pricing have also led to confrontation. Some manufacturers have refused price cuts so that 11 commonly used drugs including glibenclamide, propranolol, and frusemide will be replaced in the scheme by generic drugs. Yet the local generic companies may not be able to maintain adequate supplies; in addition, many patients will be confused by the different product names during the changeover. Another concern arising from the disagreement with the manufacturers is that some manufacturers may not request listing of important new drugs while they remain under patent and charge considerably higher prices for them outside the scheme.

This change in policy may thus destroy the scheme which has been a crucial part of health care in Australia, in that it provides the best drugs at an affordable price to everyone. The government, the doctors, and the industry must all recognise that each has legitimate concerns and that the community needs them to work together. The government needs to recognise that doctors have played an important part in the success of the drug scheme; that the alternatives to drug care are very expensive; that new drugs have the potential to reduce substantially the costs of medical treatment, reduce investigations, and prevent illness; and that more teaching in therapeutics for undergraduates and prescribing doctors is needed. At the same time industry and the Australian Medical Association must recognise that the government needs support in implementing politically unpopular measures to contain costs.

One approach to preserve the present system would be to maintain the current charges for the existing list of drugs and introduce an additional charge for new drugs. This would identify the cost of a new treatment to the patient and the doctor. For the scheme to be successful the community needs to be aware that the new drugs are expensive and that the extra cost will be subsidised-but at a premium. This message will have considerably more impact if it has the support of both the government and the Australian Medical Association.

The alternative is to restrict the prescribing of new drugs, which, inevitably, will lead to a two tier system in which disadvantaged patients are denied access to the best care - this is not acceptable.

Professor of Clinical Pharmacology and Therapeutics,

W J LOUIS

University of Melbourne,

Austin Hospital,

Heidelberg, Victoria 3084

Australia

I Scott C, Fougere G, Marwick J. Choices for health care. Report of the health benefits review. Wellington, New Zealand: New Zealand Government Printer, 1986:27.

\title{
Hip fractures in the elderly: beyond the metalwork
}

\section{Cooperation between orthopaedic surgeons and geriatricians should pay off}

Proximal femoral fracture is becoming commoner because its age specific incidence is rising in the growing numbers of the very old. ${ }^{12}$ Orthopaedic units, already experiencing difficulties in caring for their elderly patients who have been injured, may expect to admit a lot more over the next few decades, many of them even older and frailer. ${ }^{3}$ Such patients will stay longer and are more likely to require continuing care. ${ }^{4}$ Already elective orthopaedic services are under threat because of pressure on beds from patients who have been injured, ${ }^{5}$ and there is much worse to come. A large Australian study predicts an $83 \%$ increase in bed occupancy due to proximal femoral fracture between 1986 and $2011 .^{3}$

Early surgical intervention by means of internal fixation or arthroplasty is of proved value. But the wide variety of available devices-more than 100 are on the marketsuggests that limits unrelated to mere metalwork are now being encountered. "The fixations are fine," an exasperated orthopaedic surgeon remarks. "It's the bone that's the problem." Other problems that frustrate a good outcome from technically competent orthopaedic surgery for proximal femoral fracture in the elderly include poor mental state, coexistent non-surgical illness, disuse changes related to previous immobility, and social circumstances that were precarious even before the injury.

So there is more to this than orthopaedic surgery. Michael Devas, who with his geriatrician colleague R E Irvine, did so much in Hastings to pioneer the combined geriatric and orthopaedic approach, asserted that the operation is only an incident in rehabilitating the elderly patient with an orthopaedic problem. Since then several other schemes of combined care have been described,,$^{6-8}$ but without rigorous prospective assessment their value has been questioned. ${ }^{9}$

Two randomised trials of rehabilitation after proximal femoral fracture have recently been published. Gilchrist et al compared the performance of an orthopaedic geriatric unit with a traditional consultation service for patients in orthopaedic beds. ${ }^{10}$ Using no additional resources, they succeeded in recognising and treating more medical problems but failed to show any significant impact on mortality, length of stay, or eventual placement. Kennie et al reported more 
encouraging experience by comparing routine orthopaedic care, mainly in the acute ward, with geriatrician led care in a small rehabilitation unit with a strong multidisciplinary emphasis. " Some additional costs, not detailed, seem to have been incurred. There were impressive differences in the length of stay, the performance in activities of daily living, and the nature of eventual placement.

The acute orthopaedic ward is a hostile environment for frail elderly patients. In the study by Gilchrist et al transfer to routine or combined care elsewhere took place rather late, around 10 days after admission. ${ }^{10}$ By this time substantial rehabilitation opportunities might have been missed in both groups. In the study by Kennie et al the acute ward served as the control for the duration of the study, probably enhancing the effect of any alternative. Also in this study the inclusion of a scale to measure activities of daily living, a more sensitive outcome measurement than others used, seems to indicate a stronger emphasis on the potential of multidisciplinary rehabilitation. These and other minor differences between the studies might account for their conflicting results.

Geriatric orthopaedic rehabilitation is complex and requires detailed attention to patients' motivation as well as to their clinical and functional problems. And even before patients are considered the problems of logistics, the organisation of staff, and the relation between two very different specialties need to be solved. It follows that evaluating such an endeavour is much less straightforward than evaluating a pill or a procedure.

Others should be encouraged both to take on the task of rehabilitating elderly patients with orthopaedic problems and to measure the impact of their efforts as they do so. Because both orthopaedic and geriatric units vary widely in their resources, working practices, and enthusiasm for the task the nature of cooperation in different centres will vary enormously. But as the demographic imperative is potentially overwhelming and vigorous rehabilitation programmes offer the only hope of substantial reduction of hospital stay all units caring for elderly patients who have been injured now have to try.

Senior Lecturer in Geriatric Medicine,

COLIN T CURRIE

City Hospital, Edinburgh EH10 5SB

\footnotetext{
1 Wallace WA. The increasing incidence of fractures of the proximal femur: an orthopaedic epidemic. Lancet 1983;i:1413-4.

Currie AL, Reid DM, Brown N, Nuki G. An epidemiological study of fracture of the neck of femur. Health Bull (Edinb) 1986;44:143-8.

Lord SR, Sinnett PF. Femoral neck fractures: admissions, bed use, outcome and projections. Med J Aust 1986;145:493-6.

Whitaker JJ, Currie CT. Elderly orthopaedic patients: where they come from and where they go? Health Bull (Edinb) 1988;46:98-104.

Working Party of the Secretary of State for Social Services (Duthie RB, chairman). Report. Orthopaedic services: waiting time for out-patient appointments and inpatient treatment. London Orthopaedic service

6 Boyd RV, Hawthorne J, Wallace WA, Worlock PH, Crompton EH. The Nottingham orthogeriatric unit after 1000 admissions. Injury 1983;15:193-6.

7 Burley LE, Scorgie RE, Currie CT, Smith RG, Williamson J. The joint geriatric orthopaedic service in South Edinburgh. Health Bull (Edinb) 1984;42:133-40.

8 Murphy PJ, Rai GS, Lowy M, Bielswska C. The beneficial effects of joint orthopaedic-geriatric rehabilitation. Age Ageing 1987;16:273-8.

9 Gilchrist WJ, Newman RJ, Hamblen DL, Williams BO. Combined orthopaedic-geriatric care. Lancet $1985 ; \mathrm{i}: 349$.

10 Gilchrist WJ, Newman RJ, Hamblen DL, Williams BO. Prospective randomised study of an orthopaedic geriatric inpatient service. Br Med f 1988;297:1116-8.

11 Kennie DC, Reid J, Richardson IR, Kiamari AA, Kelt C. Effectiveness of geriatric rehabilitative care after fractures of the proximal femur in elderly women: a randomised clinical trial. $\mathrm{Br} \mathrm{Med} \mathcal{f}$ 1988;297:1083-6.
}

\section{Fibromyalgia syndrome: new research on an old malady}

\section{A functional syndrome that usually includes pain, fatigue, and disturbance of sleep}

Fibromyalgia is probably as old as mankind. Certainly, musculoskeletal aches and pains in the absence of arthritis, usually called "muscular rheumatism," have been well documented in Europe since the seventeenth century. ${ }^{1}$ In 1904 Sir William Gowers introduced the term fibrositis to describe muscular rheumatism of the back, ${ }^{2}$ and since then nonspecific musculoskeletal pain, usually localised, has been described under names such as fibrositis, myofascial pain, and fibromyositis. ${ }^{13}$ Nevertheless, not until 1968 was the term fibrositis used to describe a well defined syndrome with generalised musculoskeletal aching, stiffness, fatigue, poor sleep, and tenderness at some characteristic sites. ${ }^{45}$

The term fibromyalgia is now preferred to fibrositis, ${ }^{67}$ given that there is no inflammation ${ }^{89}$ and that the term fibrositis had been used previously to describe non-specific aching from heterogeneous causes..$^{1-3}$ Particular attention has recently been paid to the primary fibromyalgia syndrome, defined as non-articular rheumatism with widespread and chronic musculoskeletal aching or stiffness associated with soft tissue tenderness at multiple, characteristic sites in the absence of an underlying cause. ${ }^{+8}$ Surprisingly, in view of the common frequency and symptoms of much pain and dysfunction, ${ }^{1011}$ the first detailed and controlled study of the clinical characteristics of this syndrome was not published until $1981 .{ }^{8}$ Nevertheless, the features are now well recognised, the most important and common being generalised pain, fatigue, and disturbance of sleep. ${ }^{+811-14}$ Commonly associated symptoms include headaches, irritable bowel disturbances, a subjective sense of swelling in articular and periarticular areas, paraesthesiae, and anxiety. Over four fifths of patients are women, usually aged between 20 and 55, though the syndrome is found in children ${ }^{15}$ and the elderly. ${ }^{11}$ The important abnormality on physical examination is the presence of multiple and consistent tender points, and the results of the usual laboratory tests are normal. Positive results on testing for antinuclear antibody in the absence of a known cause have been found in $10-15 \%$ of cases, ${ }^{16}{ }^{17}$ but controlled studies are lacking. The above clinical features have been reported consistently from geographically disparate centres. ${ }^{5812-1418}$

The underlying mechanisms of the primary fibromyalgia syndrome are not well understood. Light and electron microscopic findings in muscle biopsy specimens are normal, ${ }^{9} 19$ although there has been evidence of muscle fibres being connected by a network of reticular fibres, ${ }^{20}$ and of decreased adenosine diphosphate and adenosine triphosphate concentrations, possibly owing to local hypoxia. ${ }^{21}$ Other studies have shown IgG deposition at the dermal-epidermal junction, probably indicating increased vascular permeability, ${ }^{22}$ and increased serotonin receptor binding on platelets. ${ }^{23}$ Serotonin is a modulator of pain and sleep, and its serum concentrations are significantly lower in patients with primary fibromyalgia syndrome than in normal controls ( I J Russell, personal communication).

Hence probably multiple factors, including non-restorative sleep, ${ }^{24}$ physical or mental stress, lack of muscle conditioning, or undefined constitutional factors amplify or contribute to the pain in the fibromyalgia syndrome. ${ }^{2}$ Psychological abnormalities have not been found significantly more commonly in patients seen in a general medicine outpatient 